\title{
DNA recovery from latent fingermarks treated with an infrared fluorescent fingerprint powder
}

Abdulrahman al Oleiwi ${ }^{a}$, Imtiaz Hussain ${ }^{a}$, Allyce McWhorter ${ }^{c}$, Raul Sutton ${ }^{a}$, Roberto S.P. King ${ }^{b} *$

${ }^{a}$ Faculty of Science and Engineering, University of Wolverhampton, Wulfruna Street, WV1 ILY Wolverhampton, $U K$

${ }^{b}$ Foster + Freeman Ltd., Vale Park, Evesham, Worcestershire WR11 ITD, UK

${ }^{c}$ Foster + Freeman Ltd., 46030 Manekin Plaza, No. 170, Sterling, VA 20166, USA

\section{Abstract}

The effect of the infrared fluorescent fingermark visualisation powder, $f p$ Natural $1^{\mathrm{TM}}$, on the recovery of both the quantity and quality of touch DNA from fingerprints deposited on glass slides, was investigated using qPCR and STR typing. Four donors each deposited replicate marks, which were either left untreated $(\mathrm{n}=5)$ or treated by dusting with $f p$ Natural $1^{\mathrm{TM}}(\mathrm{n}=5)$. Each sample was swabbed using the double swab technique, before being extracted using the EZNA Forensic DNA kit and then DNA quantitated before being subjected to DNA profile analysis. Results showed that there was no significant effect of $f p$ Natural $1^{\mathrm{TM}}$ on either the quantity or quality of recovered DNA. This suggests that $f p$ Natural $1^{\mathrm{TM}}$ may prove a good choice of powder for regular use at crime scenes or in the laboratory. The $f p$ Natural $1^{\mathrm{TM}}$ properties of low density, water immiscibility and low DNA affinity may account for these positive outcomes. 


\section{Keywords}

Touch DNA, $f p$ Natural 1, DNA recovery, STR typing, infrared, latent fingerprint

\section{Introduction}

Fingermarks have been used for over 100 years as a means of identifying individuals involved in crime, by virtue of the patterns deposited at crime scenes or on items of evidential value [1]. In 1997, van Oorschot et al. [2] demonstrated that fingermark residues also provide enough DNA for the generation of DNA profiles. Technological and scientific advances have improved the ability to obtain at least partial DNA profiles from evidence handled by an individual, primarily through the increased sensitivity in DNA typing procedures. DNA recovered from handled items is commonly referred to as touch DNA [3]. Sweat, oil, and exfoliated skin cells originating from the fingertips and transferred to the surface of an object may be collected and processed for DNA [4]. Conventional approaches used to recover DNA from handled evidence is known to interfere with, and often lead to the damage of, any fingermarks present on the sample in question. Accordingly, the forensic workflow usually requires exhibits to undergo fingermark visualisation processing prior to any DNA recovery and screening.

A wide variety of fingermark visualisation techniques are available, with the choice of method dependent on the type of substrate being treated or suspected fingermark composition (oil or blood contaminants, for example). Powder dusting is a common scene-based and laboratory visualisation technique that exploits the adherence of fine particulate materials to deposited fingermark residues (usually the sebaceous oils). Accordingly, a broad variety of fingerprint powders exist, that possess a range of different colors and optical properties. Previous studies have shown that different powders may be employed without significantly adversely affecting the ability of recovered DNA to be profiled [5-8], although quantitation of recovered DNA was not presented by these researchers. In contrast, others [9] have shown that some MAGNA ${ }^{\mathrm{TM}}$ jet black powder inhibits DNA IQ ${ }^{\mathrm{TM}}$ chemistry. 
One powder that has not been studied thus far, given its infancy, is the infrared (IR) fluorescent fingerprint powder recently reported by King et al. [10]. This material comprises finely milled spirulina platensis, a naturally occurring and non-toxic algae which contains components capable of near-infrared (NIR) fluorescence within its matrix. This IR fluorescent powder is suitable for use on both non-porous and semi-porous smooth substrates, and is excited with blue (420-470nm) or red (600-650nm) wavelengths, inducing strong fluorescence in the NIR region of the electromagnetic spectrum (700-850nm) (Figure 1). The use of an infrared fluorescent fingerprint powder provides great benefit to the forensic investigator given its ability to fluoresce at much longer wavelengths than conventional fingerprint powder/treatments. Accordingly, background fluorescence is regularly supressed which allows high contrast fingermarks to be observed against a background which typically 'drops-out' or become invisible within the NIR part of the spectrum. Notoriously troublesome or problematic backgrounds, such as those that are highly patterned and/or coloured, benefit most from treatment using an infrared fluorescent fingerprint powder as the visual disturbance is easily overcome, thereby allowing efficient interpretation of the friction ridge flow or ridge details to be recorded [10]. Herein, we report the timely and germane assessment into the compatibility of such a powder in relation to DNA typing procedures.

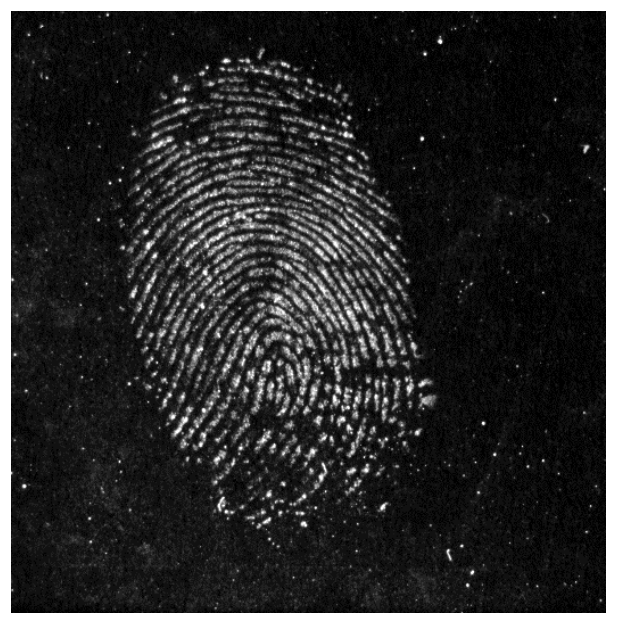

Figure 1. A latent fingermark on a polymer banknote that has been treated with $f p$ Natural $1^{\mathrm{TM}}$ fingerprint powder and visualised under 600-650nm illumination with $715 \mathrm{~nm}$ long-pass camera filter using a Foster + Freeman Crime-lite Imager. 


\section{Methods and Materials}

Deposition of fingermarks: $76 \times 26 \mathrm{~mm}, 0.8$ to $1.0 \mathrm{~mm}$ thick Glass slides (Fisher Scientific FB58620, UK) were cleaned following the method described by Oleiwi et al. [11]. One to two hours after arriving at work (between 10-11 am, at the same time each day for each donor), four participants, without any explicit instructions as to behaviour, including, for example, hand washing, deposited combined middle and ring fingermarks onto a cleaned glass slide. Samples were collected by pressing both finger tips for a few seconds onto the glass slide. This process was repeated once daily until the required number of samples were accumulated. 12 slides were allocated to each participant: 5 were treated with $f p$ Natural $1^{\mathrm{TM}}$ powder (powder visualised) with each donor allocated a separate brush, 5 were left untreated and served as positive controls (untreated fingermarks), and 2 were negative controls (no fingermark but processed as for fingermarks). At the end of sample collection, slides were stored for two weeks in a plastic slide box at $4^{\circ} \mathrm{C}$ which had been washed in the same way as the slides.

Collection of DNA: The double swab method was used, consisting of swabbing the identified area with a DNA-free sterile cotton ear bud, UV irradiated for 15 mins, which was moistened with filtered distilled water $(50 \mu \mathrm{l})$ before swabbing the same area with a dry swab [12]. $f p$ Natural $1^{\text {TM }}$ powder was examined for any human DNA background by dipping a moistened cotton bud into powder. Then the $f p$ Natural $1^{\text {TM }}$ loaded bud underwent DNA extraction. If the $f p$ Natural $1^{\mathrm{TM}}$ powder tested positive for DNA, the treated samples would have been discarded.

DNA extraction and Quantification: DNA was extracted from swabbed samples using the E.Z.N.A.® Forensic DNA Kit (Omega Bio-Tek), following the standard protocol described in the manufacturer's instructions. The extracted DNA was eluted with $50 \mu 1$ to maximise DNA yields. $2 \mu \mathrm{l}$ duplicates of each sample's extracted DNA was quantified using the Investigator® Quantiplex Quantification Kit assay (QIAGEN, Crawley, UK). The quantification process was carried out following the manufacturer's instructions, using an ABI® 7500 Fast Real-Time PCR System (Applied Biosystems, USA). Results were analysed using SDS 1.9.1 software (Applied Biosystems, USA) and interpretation was based on criteria recommended by the kit manufacturer. 
Profiling of DNA samples: Samples were amplified using a PowerPlex ${ }^{\circledR}$ ESI 16 Fast System (Promega, USA) for 30 cycles, following the manufacturer's 'amplification of extracted DNA' protocol. DNA samples were, when needed, diluted to obtain a maximum concentration of $0.5 \mathrm{ng} / \mu 1.5 \mu \mathrm{l}$ of DNA sample was added to $20 \mu \mathrm{l}$ of the amplification reaction mix using the GeneAmp® PCR System 9700 (Applied Biosystems, USA). PCR products were subjected to electrophoresis using an ABI 3500 Genetic Analyser (Applied Biosystems, USA) and profiles were analysed using GeneMapper® ID v3.2 Software (Applied Biosystems, USA). The criteria used to estimate a peak as an allele are the same as those published in Oleiwi (2015) [13]. A minimum peak threshold of 50 relative fluorescent units (RFUs) was applied. A negative control was used for each STR amplification batch and, if any sample had shown positive, would have resulted in the entire batch being disregarded.

Statistical analysis: Statistical analysis of data was performed using GraphPad Prism version 7.0 for Windows, (GraphPad Software, La Jolla California, USA).

\section{Results and discussion}

The results showed that all negative controls yielded no recoverable DNA, confirming the care in removing contaminating DNA from the experimental areas. Both positive controls and test sample data is shown in Table 1 and figure 2.

\begin{tabular}{|c|c|c|c|c|c|c|c|c|c|}
\hline \multirow[t]{2}{*}{ Donor } & \multicolumn{4}{|c|}{ Untreated (ng DNA) } & \multicolumn{4}{|c|}{$f p$ Natural $1^{\mathrm{TM}}$ Treated (ng DNA) } & \multirow[t]{2}{*}{$P$ value } \\
\hline & Mean & SD & $\begin{array}{c}\text { Lowest } \\
\text { DNA } \\
\text { amount }\end{array}$ & $\begin{array}{c}\text { Highest } \\
\text { DNA } \\
\text { amount }\end{array}$ & Mean & SD & $\begin{array}{c}\text { Lowest } \\
\text { DNA } \\
\text { amount }\end{array}$ & $\begin{array}{c}\text { Highest } \\
\text { DNA } \\
\text { amount }\end{array}$ & \\
\hline 1 & 1.52 & 0.49 & 0.3 & 1.9 & 1.51 & 1.05 & 0.4 & 1.75 & $>0.9999$ \\
\hline 2 & 0.91 & 0.48 & 0.2 & 1.3 & 0.87 & 0.53 & 0.1 & 1.0 & $>0.9999$ \\
\hline
\end{tabular}




\begin{tabular}{|l|c|c|c|c|c|c|c|c|c|}
\hline 3 & 0.85 & 0.71 & 0.1 & 1.0 & 0.62 & 0.27 & 0.15 & 0.8 & 0.9823 \\
\hline 4 & 0.97 & 0.35 & 0.25 & 1.5 & 1.01 & 0.25 & 0.4 & 1.0 & $>0.9999$ \\
\hline
\end{tabular}

Table 1. The recovery of DNA from $f p$ Natural $1^{\mathrm{TM}}$ treated fingermarks and untreated

fingermarks from 4 donors. The table shows mean and standard deviation for 5 events for each donor.

Table 1 shows individual means of event data for the treated and untreated replicates. It can be seen that the recovered amount of DNA was highly variable, as standard deviations varied from greater than, to about one third of, the mean values. The high similarity between the mean values of untreated and treated samples were tested using the Student $t$-test, and in all cases showed no significant difference between test and control samples. This data, cumulated and depicted in Figure 2, illustrates the mean quantity of DNA recovered from untreated and $f p$ Natural $1^{\mathrm{TM}}$ treated samples, where means were 1.1 and 0.99 , respectively. The difference in the cumulated data was statistically not significant with a $p$ value of 0.38 when assessed using an unpaired ttest.

The $\mathrm{C}_{\mathrm{T}}$ values of IPC ranged between 30.01 and 31.87 indicating no inhibition of real time PCR amplification was observed in any of the samples, as $\mathrm{C}_{\mathrm{T}}$ values were all within the optimum range of $31 \pm 1$

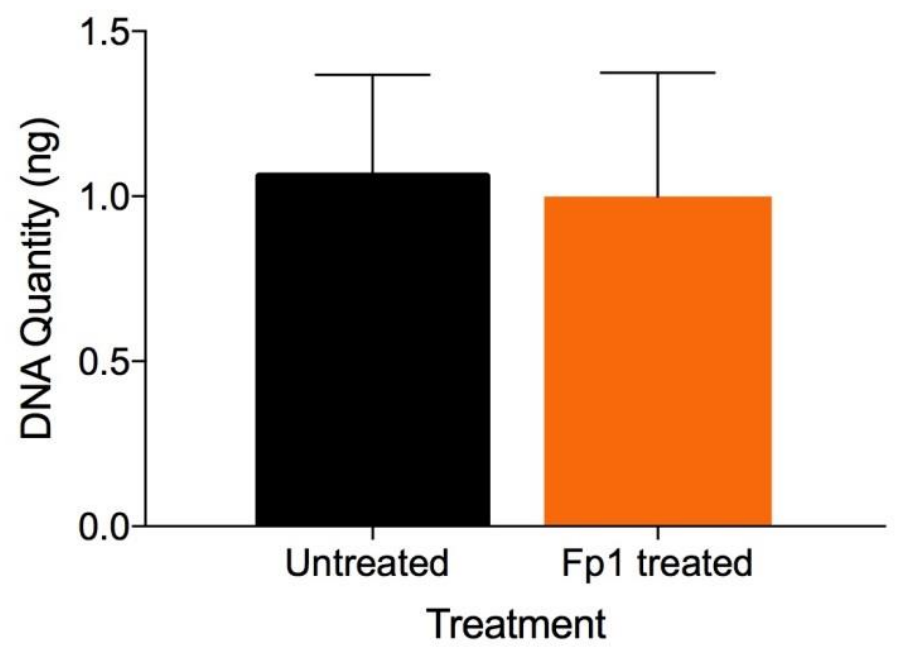

Figure 2. Cumulated DNA quantification data for fingermarks treated with $f p$ Natural $1^{\mathrm{TM}}$ when compared to untreated controls 
The threshold cycle $\left(\mathrm{C}_{\mathrm{t}}\right)$ values for the Internal PCR Control (IPC) were in the range of 28-30 for all samples, meaning that PCR inhibition was not indicated during quantification of the samples (Table 1). Human quantification values were obtained for a majority of the samples tested, and range, average, and standard deviation values are recorded in Table 1.

The quality of the recovered human DNA samples was assessed by DNA profiling the fingermark extracts. An example of a typical DNA profile is shown in Figure 3. The cumulated results of this analysis are shown in Table 2. A paired $t$-test shows that differences in numbers of alleles called comparing treated and untreated fingermarks was non-significant with $p=0.21$. In the experimental protocol used in this series of experiments, donor DNA profiles showed a small number of non-donor allele contamination. For samples that were not powder treated, out of 20 donor profiles analysed four non-donor called alleles were found compared with 2 in the powder treated set of 20 .

Figure 3. A typical electrophoretogram for DNA from recovered fingermarks. DNA was extracted as described in the materials and methods. The profile was obtained using PowerPlex® ESI 16 Fast System (Promega, USA) for 30 cycles. This profile is representative of samples that contained enough DNA to yield profiles, showing minor stutter peaks and minor drop in at individual loci.

A small number of called alleles not originating from donors were seen in these samples, but does not compare with the donor contamination levels described elsewhere [14]. These authors ascribe differences to hand washing process used in [11]. However, the results presented herein indicate a lower level of alleles that do not result from the donor of the fingermark. A possible explanation for this may relate to the time for sampling. This was approximately one to two hours after arrival at work. All of the donors drove to work, and will normally use bathroom facilities in that first hour, followed by washing of hands with liquid soap. This was followed by work at a sole user desk, where levels of contaminant DNA would have been at a minimum.

\begin{tabular}{|c|c|c|c|c|c|c|}
\hline \multicolumn{7}{|c|}{ Number of called Alleles } \\
\cline { 2 - 7 } & $\mathbf{0}$ & $<10$ & $\geq 10$ & $>\mathbf{2 0}$ & $\geq \mathbf{3 0}$ & Extra allele \\
\hline
\end{tabular}




\begin{tabular}{|c|c|c|c|c|c|c|}
\hline Untreated & 2 & 12 & 5 & 0 & 1 & 4 \\
\hline Treated & 2 & 14 & 4 & 0 & 0 & 2 \\
\hline
\end{tabular}

Table 2. Comparison of untreated and $f p$ Natural $1^{\mathrm{TM}}$ treated fingermarks for the recovery of DNA by examination of profiles generated using a PowerPlex® ESI 16 Fast System for called alleles. The number of alleles that did not originate from the donor is shown in the right hand column.

\section{Conclusion}

$f p$ Natural $1^{\mathrm{TM}}$ infrared fluorescent fingerprint powder was shown to have a statistically insignificant effect on the quantity of touch DNA recovered, when measured by qPCR. This is supported by analysis of the cumulated profile data generated from the extracted DNA. The number of called alleles was not statistically significantly different in either the treated or untreated DNA extracts of fingermark residues.

Visual inspection of extraction tubes after centrifugation showed two points of interest. Firstly, the particles of fingerprint powder floated on top of the extraction medium as a green flotate. Secondly, there appeared to be no leaching of any dyestuffs from the particle into the extraction medium. The small loss of DNA also indicated little adherence of DNA to the powder fragments. $f p$ Natural $1^{\mathrm{TM}}$ has these three properties that may make it an ideal fingermark visualisation reagent to use in combination with DNA profile analysis: particle density is lighter than water, so particles float on solution surfaces and do not contaminate subsequent DNA profiles; the powder itself is water insoluble, and so does not transfer to the PCR amplification stage, and the powder has little affinity for DNA, meaning losses due to powder binding are minimized. We can draw some inferences for those developing new fingermark visualisation powders, if DNA recovery is a consideration, in particular reagent density, miscibility in water and DNA affinity could be part of the considerations in design. An analysis of allele recovery from STR profiles could be generated from fingermarks treated with $f p$ Natural $1^{\text {TM }}$ which showed little difference to untreated fingermarks. Although untreated latent fingermarks resulted in a higher human quantification and RFU values than samples treated with the powder alone. As shown in previous research [10], the inherent properties of the IR fluorescent fingerprint powder 
allows for extraordinary contrast in samples that would otherwise be very difficult to detect and treat for fingerprints; thereby accentuating its potential within the forensic practitioners arsenal. The ability to still effectively recover DNA from samples treated with this IR fluorescent powder highlights the minimally invasive nature of this particular fingerprint visualisation process, which when coupled with its inherent optical properties, provides the investigator with an extremely powerful tool that may be added to the forensic workflow.

\section{Acknowledgements}

We thank CARA for support to Abdulrahman Oleiwi and the University of Wolverhampton for providing the laboratory in order to carry out the investigations. We acknowledge the help of fingermark donors used throughout this study.

\section{References}

[1] Barnes J G "History" in McRoberts A (2011) Fingerprint Source Book, chapter 1

[2] van Oorschot, R.A.H. and Jones, M.K. (1997) DNA Fingerprints from Fingerprints. Nature, 387(6635), pp. 767

[3] Butler J.M., "Low-level DNA testing: issues, concerns, and solutions", in Advanced topics in forensic DNA typing: methodology, 311-341. Waltham, MA: Elsevier, 2012.

[4] Wickenheiser R.A., Trace DNA: a review, discussion of theory and application of the transfer of trace quantities of DNA through skin contact, J. Forensic Sci. 47(3) (2002) 442-450.

[5] C. Thamnurak, W. Bunakkharasawat, S. Riengrojpitak, N. Panvisavas, DNA typing from fluorescent powder dusted latent fingerprints, Forensic Sci. Int.: Genetics Supplement Series 3 (2011) e524-e525.

[6] D.E.O. Van Hoofstat, D.L.D. Deforce, I.P. Hubert De Pauw, E.G. Van den Eeckhout, DNA typing of fingerprints using capillary electrophoresis: effect of dactyloscopic powders, Electrophoresis 20 (1999) 2870-2876. 
[7] J.J. Raymond, C. Roux, E.D. Pasquier, J. Sutton, C. Lennard, The effect of common fingerprint detection techniques on the DNA typing of fingerprints deposited on different surfaces, J. of Forensic Identif. 54 (1) (2004) 22-44.

[8] M. Pesaresi, L. Buscemi, F. Alessandrini, M. Cecati, A. Tagliabracci, Qualitative and quantitative analysis of DNA recovered from fingerprints, Int. Congress Series 1239 (2003) 947-951.

[9] Laurin, N; Célestin, F.; Clark, M.; Wilkinson, D.; Yamashita, B.; Frégeau C. New incompatibilities uncovered using the Promega DNA IQ ${ }^{\mathrm{TM}}$ chemistry For. Sci. Int. 257 (2015) 134-141

[10] R.S.P. King, P.M. Hallett, D. Foster, Seeing into the infrared: a novel IR fluorescent fingerprint powder, Forensic Sci. Int. 249 (2015) e21-e26.

[11] Oleiwi, A., Schmerer, W.M., Morris, M. R., Sutton R. The relative DNA-shedding propensity of palmar and fingerprint surfaces, Science and Justice 55, (2015) 329-334.

[12] D. Sweet, M. Lorente, J.A. Lorente, A. Valenzuela, E. Villanueva, An Improved Method to Recover Saliva from Human Skin: The Double Swab Technique, J Forensic Sci. 42 (1997) 320-322.

[13] Oleiwi (2015) Experimental approached to improving DNA recovery from developed fingerprints, $\mathrm{PhD}$ thesis, University of Wolverhampton.

[14] Goray, M., Fowler, S., Szkuta, B., van Oorschot R.A.H. Shedder status-An analysis of self and non-self DNA in multiple handprints deposited by the same individuals over time. Forensic Sci. Int.: Genetics 23 (2016) 190-196. 